consumption have been developed for middle-aged men. These were mainly delivered in healthcare settings. Disadvantaged young men seldom attend healthcare. A tailored brief intervention delivered by text message provides a low cost method for accessing this hard to reach group.

Methods Focus groups were conducted with disadvantaged young men to explore the social context of drinking and opportunities for intervention. Empirical evidence and social cognition theories were used to develop a series of text messages and images to reduce binge drinking. These were tested in three further focus groups, including one with female partners of binge drinkers.

Results The focus groups identified cost and personal experience of alcohol harms as opportunities for intervention. Humour was identified as a useful lever for behaviour change. The focus groups also provided useful quotes for text messages. A series of 37 text messages and images were developed within the framework of the Stages of Change model. They incorporated the principles of motivational interviewing and used components of successful behaviour change strategies. To promote interaction, several messages requested a response.

Conclusion This study has shown it is possible to encapsulate the theoretical approaches and the major components of brief interventions in a series of text messages. Focus groups highlighted levers for change and aided the selection of acceptable messages. This approach could be used with other social groups and other adverse health behaviours.

\section{P2-53 YOUNG ADULT LUNG FUNCTION AND CAROTID INTIMA- MEDIAL THICKNESS}

doi:10.1136/jech.2011.142976h.88

B Curry,* A Venn. Menzies Research Institute, University of Tasmania, Hobart, Australia

Introduction Impaired lung function has been associated with an increased risk of cardiovascular disease outcomes, mortality and subclinical disease including increased carotid intima-media thickness (cIMT) and carotid plaques. However few population-based studies have investigated the association between lung function and cIMT in young adults.

Objective To investigate the associations of lung spirometric measures with cIMT in healthy young Australian men and women.

Methods Data were collected from a population-based sample of 872 males and 792 non-pregnant females (aged 26-36 years). The associations of adult Forced Vital Capacity (FVC) and Forced Expiratory Volume $\left(\mathrm{FEV}_{1}\right)$ with cIMT were investigated using partial correlation.

Results The mean cIMT values (SD) were 0.581 (0.099) and 0.553 (0.080) $\mathrm{mm}$ for non-smoking, and 0.575 (0.097) and 0.567 (0.099) $\mathrm{mm}$ for smoking males $(\mathrm{n}=187)$ and females $(\mathrm{n}=161)$ respectively. Significant negative associations of $\operatorname{FEV}_{1}(\rho=-0.100 \mathrm{p}=0.012)$ and FVC $(\rho=-0.087 \mathrm{p}=0.029)$ with cIMT were observed for nonsmoking females after adjustment for age, height, asthma, blood pressure, weight, percentage body fat and fitness. In males there was also a negative association of FVC with cIMT in non-smokers $(\rho=-0.076 \mathrm{p}=0.058)$ and with $\mathrm{FEV}_{1} / \mathrm{FVC}$ in smokers $(\rho=-0.196$ $p=0.009)$. These associations were independent of $C$ reactive protein and blood lipids. No significant associations were observed for female smokers.

Conclusion In this sample of healthy young men and women airway obstruction was associated with higher cIMT in male smokers. However in non-smoking men and women, FVC was also associated with higher cIMT independently of other cardiovascular disease risk factors.

\section{P2-54 DIET AND RISK OF DIVERTICULAR DISEASE IN THE EUROPEAN PROSPECTIVE INVESTIGATION INTO CANCER AND NUTRITION (EPIC)-OXFORD COHORT, A PROSPECTIVE STUDY OF BRITISH VEGETARIANS AND NON-VEGETARIANS}

doi:10.1136/jech.2011.142976h.89

F Crowe, ${ }^{*}$ P Appleby, N Allen, T Key. University of Oxford, Oxford, UK

Introduction Vegetarians and those consuming a high fibre diet are thought to have a lower risk of diverticular disease but there is little evidence from prospective studies to substantiate these associations. The objective was to examine the associations between vegetarianism and dietary fibre intake with the risk of diverticular disease in the EPIC-Oxford study.

Methods This analysis of the EPIC-Oxford cohort included 47033 men and women living in England or Scotland of whom 15459 (33\%) were vegetarians. Diverticular disease cases were identified through linkage with hospital records. The RR of diverticular disease by vegetarian status and quintiles of dietary fibre intake was estimated using multivariate Cox proportional hazards regression models.

Results After an average follow-up of 11.6 years, there were 812 incident cases of diverticular disease. Vegetarians had a $30 \%$ lower

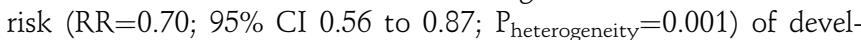
oping diverticular disease compared with non-vegetarians. There was also an inverse association with fibre intake; participants in the highest quintile ( $>25 \mathrm{~g} /$ day) of dietary fibre intake had a $41 \%$ lower risk $\left(\mathrm{RR}=0.59 ; 95 \% \mathrm{CI} 0.46\right.$ to $\left.0.78 ; \mathrm{P}_{\text {trend }}<0.001\right)$ compared to those in the lowest quintile ( $<14 \mathrm{~g} /$ day). After mutual adjustment, both a vegetarian diet $\left(P_{\text {heterogeneity }}=0.015\right)$ and a higher intake of fibre $\left(\mathrm{P}_{\text {trend }}=0.001\right)$ were significantly associated with a lower risk of diverticular disease.

Conclusion Consuming a vegetarian diet and a high intake of dietary fibre were both associated with a lower risk of diverticular disease.

\section{P2-55 INFLUENCE OF SEXUAL MATURATION ON BODY MASS INDEX IN A LONGITUDINAL STUDY WITH ADOLESCENTS FROM BRAZIL}

doi:10.1136/jech.2011.142976h.90

${ }^{1} \mathrm{D}$ Cunha, ${ }^{2} \mathrm{G}$ Veiga, ${ }^{1} \mathrm{R}$ Sichieri. ${ }^{1}$ Institute of Social Medicine, State University of Rio de Janeiro, Rio de Janeiro, Rio de Janeiro, Brazil; '2Department of Nutrition, Federal University of Rio de Janeiro, Rio de Janeiro, Rio de Janeiro, Brazil

Introduction Body mass index (BMI) during adolescence is highly influenced by the pubertal maturation, and early maturation has been associated with overweight, but it is still uncertain if these factors are just a cluster of conditions or whether pubertal maturation influence BMI increments. This study aimed to examine the influence of sexual maturation (SM) on fatness and BMI increments during adolescence among Brazilians aged between 9 and 13 years. Methods 598 girls and 518 boys were followed from 1-year school period. Maturational timing was self-assessed using the Tanner stage ratings (breast development for girls and pubic hair for boys). Early maturation was determined as the age bellow the median age within each gender SM group.

Results Girls with early maturation showed a statistically significant greater prevalence of overweight (WHO classification) compared to those without early maturation $(23.7 \%$ vs $15.4 ; \mathrm{p}=0.02)$ at baseline and similar results were found after 1-year follow-up. On the other hand weight gain during follow-up was not associated to maturation either calculated by stages of sexual maturation, according to Tanner staging or to early maturation, for girls and boys.

Conclusions The results suggest that at baseline and follow-up BMI was highly associated with SM for girls, but BMI increments during follow-up was unrelated to SM for both sexes suggesting a non 